\title{
Renal cell carcinoma intravascular spread into segmental branches of the renal vein: a single institution case series
}

\author{
Mauro Giuseppe Mastropasqua ${ }^{1, *}$, Marta Mariano ${ }^{1}$, Federico Fusco ${ }^{2}$, Gerardo Cazzato ${ }^{1}$, Valentina Ruggiero ${ }^{1}$, \\ Michele Battaglia ${ }^{3}$, Giuseppe Lucarelli ${ }^{3}$, Giuseppe Ingravallo ${ }^{1}$, and Eugenio Maiorano ${ }^{1}$
}

1 Department of Emergency and Organs Transplantation, Section of Anatomic Pathology, School of Medicine, University "Aldo Moro", Bari; mauro.mastropasqua@uniba.it

2 Department of Emergency and Organs Transplantation, Section of Anatomic Pathology, School of Medicine, University "Aldo Moro", Bari; martamariano05@gmail.com

3 Institute of Pathology, Technical University of Munich (TUM), Munich; federico.fusco@tum.de

4 Department of Emergency and Organs Transplantation, Section of Anatomic Pathology, School of Medicine, University "Aldo Moro", Bari; gerycazzato@hotmail.it

5 Department of Emergency and Organs Transplantation, Section of Anatomic Pathology, School of Medicine, University “Aldo Moro", Bari; valentinaruggiero.vr53@gmail.com

6 Department of Emergency and Organs Transplantation, Urology, Andrology and Kidney Transplantation Unit, University “Aldo Moro", Bari; giuseppe.lucarelli@uniba.it

7 Department of Emergency and Organs Transplantation, Urology, Andrology and Kidney Transplantation Unit, University “Aldo Moro", Bari; michele.battaglia@uniba.it

8 Department of Emergency and Organ Transplantation, Section of Anatomic Pathology, School of Medicine, University “Aldo Moro", Bari; giuseppe.ingravallo@uniba.it

9 Department of Emergency and Organ Transplantation, Section of Anatomic Pathology, School of Medicine, University "Aldo Moro", Bari; eugenio.maiorano@uniba.it

* Correspondence: mauro.mastropasqua@uniba.it Tel.: +390805593546

Simple Summary: Prognosis of renal cell carcinoma may be poor when it is diagnosed at higher stage because of its extrarenal extension (mostly to the intraparenchymal branches of the renal vein). The assessment of retrograde venous invasion may be challenging, with a high inter-observer variability. To reduce this variability, we have introduced an adjustment of the macroscopic approach to renal gross sampling. In order to demonstrate the effectiveness of this more laborious method of sampling, we have compared the pathological staging as obtained following standard and modified procedures. The results of our study have highlighted as, thanks to the modified procedure, a higher number of cases with invasion of renal vein has been detected, providing a more accurate staging of the patient that could benefit of better fitting treatments.

\begin{abstract}
Background: Overall survival of the patients with renal cell carcinoma (RCC) depends mostly on extra-renal extension, documented by the invasion of the pelvicalyceal system, or the perinephric/renal sinus fat or the renal vein/its segmental intraparenchymal branches. Staging may be challenging because of the high inter-observer variability. We have introduced a more accurate procedure to detect the extra-renal extension and, to possibly evaluate the impact of such more laborious approach, we have compared the RCC pathological staging obtained following both standard and modified procedures; (2) Methods: We selected 54 consecutive cases of RCC diagnosed 18 months before and 54 consecutive cases diagnosed 18 months after the introduction of the new method of sampling. Clinico-pathological characteristics have been statistically analyzed; (3) Results: Most of the features analyzed were non statistically significative, except the extra-renal invasion. More precisely, the occurrence of retrograde venous invasion was higher in the cases grossly approached with the more accurate method; (4) Conclusions: Extra-renal extension to intraparenchymal renal vein branches may be underestimated, leading to an inappropriate under-staging. Using more accurate staging procedures, to disclose the occurrence of intra-renal veins tumoral thrombosis, helps to better define the pathological stage, allowing patients to benefit of better fitting treatments.
\end{abstract}

Keywords: Renal Cell Carcinoma; Pathological staging; Extrarenal extension; Renal vein invasion 


\section{Introduction}

Renal cell carcinoma (RCC) is the most frequent malignant tumor of the kidney in adulthood, representing $90 \%$ of all renal tumors [1]. Overall, survival mostly depends on stage of the disease, histotype and grade, according to the recent WHO classification of tumor of the urinary system [1-3].

The pathological stage of the disease varies according to two main features: tumor size $(\mathrm{pT} 1,2)$ and extra-renal extension $(\mathrm{pT} 3 \mathrm{x})$. As specified by the $\mathrm{pT}$ suffixes, the occurrence of extra-renal extension worsens the prognosis, irrespective of tumor size, thus setting the case at a higher stage [4]. Extra-renal extension encompasses invasion of the renal vein or its segmental branches, invasion of the pelvi-calyceal system, invasion of the perirenal fat and/or renal sinus. Wider extension of disease includes invasion of the vena cava, the Gerota's fascia and ipsilateral adrenal gland involvement [1]. Obviously, such distinct $\mathrm{pT}$ stratification also implies sharp prognostic differences: 5-year overall survival of patients harboring pT1-2 tumors is $92.6 \%$, as opposed to $66.7 \%$ for pT3a [5]. Therefore, it is of paramount relevance to correctly identify and evaluate extra-renal extension, since gross examination of the surgical sample [6-8], to avoid inappropriate staging, inadequate complimentary treatments and less favorable outcome $[9,10]$.

A recent study has demonstrated high inter-observer variability as to proper RCC staging [6]. Frequently, clear cell RCC shows predilection for intravenous growth [11] and this peculiar feature may represent a source of discordance, mostly due to the underestimation of subtle finger-like protrusions extending, in a retrograde fashion, into intraparenchymal veins [6]. Intravenous spread may be misinterpreted as satellite foci of a multifocal tumor [12]. According to the recent recommendations of the International Society of Urological Pathology (ISUP) [7,8], since macroscopic handling of the surgical specimen it is mandatory to evaluate all the crucial sites which contribute to the assessment of extra-parenchymal extension. Furthermore, ISUP recommendations [8] also proposed clear cut instructions on how to correctly identify extra-parenchymal extension at a microscopic level, while well-defined guidelines to properly assess intra-parenchymal venous branches invasion are still lacking. Therefore, careful gross evaluation of renal sinuses, as well accurate search for intra-parenchymal venous spreading may result of pivotal relevance for prognostic and predictive purposes [13-15].

In order to reduce inter-operator variability, to avoid under-staging of the disease, and to more precisely define RCC patients' prognosis, we hereby propose an adjustment of the macroscopic approach to surgical samples, along with more extensive microscopic evaluation of the renal sinus. Also, to possibly evaluate the impact of such more laborious approach, we compared the RCC pathological staging as obtained following standard procedures and according to the modified procedures.

\section{Materials and Methods}

Starting on May 2017, taking into account ISUP recommendations $[7,8]$ and relevant studies on this subject, $[16,17]$ we have introduced more extensive sampling of the renal sinus for cases grossly doubtful for invasion of renal sinus, in view of the proximity of the tumor mass; in addition, we also performed immunohistochemical stains for desmin and CD31, to more accurately identify intra-parenchymal venous branches possibly invaded by the tumor.

Briefly, after removing the perinephric fat, accurate sampling of any area firmly adhering to it and inking its inner surface, according to this new procedure the renal vein was opened up to its closer internal branches present at the hilum (once the specimen had been bi-valved) and carefully examined to detect the presence of any thrombi. Subsequently, 3-5 samples from the renal sinus/parenchymal interface were obtained and submitted for histological examination.

Up to November 2018, 141 RCC cases examined according to the new procedure had accumulated and, for comparative purposes, all RCC diagnosed over the 18 months preceding the application of this procedure were collected. 
All microscopic slides were independently and blindly reviewed by three pathologist trainees (GC, MM, VR) and, in case of divergent opinions, the slides were collegially reviewed and discussed together with a senior uro-pathologist (MGM), to come to a final agreement.

The revision of each case included analyses of the following clinico-pathological characteristics: age, gender, type of surgery (radical nephrectomy vs. nephron sparing surgery) side and size of the tumor, histological subtype and grade (according to the WHO and ISUP recommendations) [1,3,19], TNM stage (according to 8 th edition) [4] and, for pT3x cases, occurrence of extrarenal extension. Cases showing neoplastic satellite nodules without clear cut evidence of intra-vascular localization were further investigated by immunohistochemistry with anti-desmin and anti-CD31 antibodies.

All data were statistically analyzed by Pearson's chi-squared test, Fisher's exact test and t-test.

Informed consent was obtained from all individual participants included in the study. All procedures were in accordance with the ethical standards of the institutional and/or national research committee and with the 1964 Helsinki Declaration and its later amendments or comparable ethical standards. The research project was approved by the local Ethics Committee (n. 143/CE/2015)".

\section{Results}

Overall, this study included 139 RCC (Group 1: November 2015 - April 2017) sampled according to standard procedures and 141 cases (Group 2: May 2017 - October 2018) sampled with the new protocol (Table 1).

Table 1. Type of surgery and cohort object of the study

\begin{tabular}{|l|l|l|}
\hline & $\begin{array}{l}\text { Group 1 } \\
\text { Nov 2015-Apr } \\
2017 \\
\mathrm{~N}(\%)\end{array}$ & $\begin{array}{l}\text { Group 2 } \\
\text { May 2017-Oct 2018 } \\
\mathrm{N}(\%)\end{array}$ \\
\hline $\begin{array}{l}\text { Partial } \\
\text { nephrectomy }\end{array}$ & 85 & 87 \\
\hline $\begin{array}{l}\text { Radical } \\
\text { nephrectomy }\end{array}$ & $54(38.9)$ & $54(38.3)$ \\
\hline Total & 139 & 141 \\
\hline
\end{tabular}

Among these, 54 cases in each group (38.9\% and 38.3\%) were obtained from radical nephrectomy and represented the object of our study; partial nephrectomy samples were excluded due to the impossibility to appropriately and systematically apply the proposed protocol.

Clinico-pathological results are summarized in table 2.

Table 2. Clinico-pathological characteristics

\begin{tabular}{|l|l|l|l|}
\hline & $\begin{array}{l}\text { Group 1 } \\
\text { Nov 2015-Apr 2017 } \\
\mathrm{N}(\%)\end{array}$ & $\begin{array}{l}\text { Group 2 } \\
\text { May 2017-Oct 2018 } \\
\mathrm{N}(\%)\end{array}$ & $p$-value \\
\hline Gender & & & $p=<0.05$ \\
\hline Males & $42(77.8)$ & $36(66.7)$ & \\
\hline Females & $12(22.2)$ & $18(33.3)$ & \\
\hline Age (ys) & 61.9 & 63.8 & $p=0.20$ \\
\hline
\end{tabular}




\begin{tabular}{|c|c|c|c|}
\hline Side & & & $p=0.50$ \\
\hline Right & $30(55.6)$ & $31(57.4)$ & \\
\hline Left & $24(44.4)$ & $23(42.6)$ & \\
\hline Size $(\mathrm{cm})$ & 5.61 & 5.29 & $p=0.50$ \\
\hline Histological subtypes [1] & & & $p=0.98$ \\
\hline CCRCC & $39(72.2)$ & $36(66.7)$ & \\
\hline PapRCC & $8(14.8)$ & $6(11.1)$ & \\
\hline ChRCC & $5(9.3)$ & $7(13)$ & \\
\hline Others & $2(3.7)$ & $5(9.2)$ & \\
\hline Grade [1] & & & $p=0.21$ \\
\hline G1 & $12(22.2)$ & $15(27.8)$ & \\
\hline G2 & $29(53.7)$ & $21(38.9)$ & \\
\hline G3 & $11(20.4)$ & $15(27.8)$ & \\
\hline G4 & $2(3.7)$ & $3(5.6)$ & \\
\hline Extrarenal extension & & & $p=0.6$ \\
\hline $\begin{array}{l}\text { Overall (perinephric fat, } \\
\text { renal sinus fat, } \\
\text { intraparenchymal } \\
\text { branches of renal vein) }\end{array}$ & $14(25.9)$ & $18(33.3)$ & \\
\hline $\begin{array}{l}\text { intraparenchymal } \\
\text { branches of renal vein }\end{array}$ & $1(1.8)$ & $9(16.7)$ & $p=0.019$ \\
\hline Total & 54 & 54 & \\
\hline
\end{tabular}

CCRCC: Clear Cell Renal Cell Carcinoma; PapRCC: Papillary Renal Cell Carcinoma; ChRCC: Chromophobe Renal Cell Carcinoma

In Group 1, 42 patients were males (77.8\%) and 12 females (22.2\%) while in Group 2 there were 36 males $(66.7 \%)$ and 18 females (33.3\%), in agreement with the literature; [1] nevertheless, a slightly lower prevalence in males was detected in Group 2 and, in comparison with Group 1, the difference was statistically significant $(\mathrm{p}=0.033)$.

The mean age at presentation was 61.9 in Group 1 and 63.8 in Group 2, without statistically significant differences ( $t$-value $=-1.27 ; \mathrm{p}=0.20$ ). The right side was the most affected in both groups: 30 cases (55.6\%) in Group 1, 31 (57.4\%) in Group 2, without statistically significant differences. The mean size of tumors in Group 1 was $5.61 \mathrm{~cm}$ (range: 1.9-22) and 5.29 in Group 2 (range: $2-23 \mathrm{~cm}$ ), in agreement with the literature [18] and without statistically significant differences $(\mathrm{p}=0.50)$ between the groups. Tumor sizes of the tumors confined to the kidney, when stratified in four classes according to TNM (pT1a,b and pT2a,b), lead to comparable results in both groups, without statistically significant differences $(\mathrm{p}=0.53)$.

In line with the literature data [1], clear cell renal cell carcinoma (CCRCC) was the prevalent histological subtype in both groups (39 case in Group 1, 36 in Group 2), followed by papillary RCC (8 cases vs. 6). Chromophobe carcinomas were slightly more in Group 2 (7 vs 5 cases), as well as other histological subtypes (5 vs 2 cases), including 2 papillary clear cell RCC, 2 cases of unclassified carcinomas with sarcomatoid features and 1 multilocular cystic renal neoplasm of low malignant potential.

No statistically significative differences were identified when stratifying the histological subtypes for gender, age ( $\mathrm{p}=0,98$ gender; $\mathrm{p}=0,37$ age) or size. 
As to tumor grade, the cases of Group 2 were more homogenously distributed in a 4-tier scale [1,19] if compared to Group 1, with G2 cases being more abundant. However, statistical analyses did not demonstrate any significant differences $(p=0.21)$ between the groups.

Overall, higher percentages of tumors with extrarenal extension (pT3x) were detected in Group 2. without statistically significant differences $(p=0.29)$. Also, extrarenal extension and tumor size and grade appeared directly correlated in both groups, once again without statical significance $(\mathrm{p}>0.16)$.

As to the occurrence of perinephric and renal sinus fat invasion, both were equally represented in the 2 groups, without any statistically significant differences $(p=0.6)$.

Notably, a relevant difference was highlighted with regard to the occurrence of invasion of the intra-parenchymal branches of the renal vein: 9 out of 54 cases $(16.7 \%)$ in the Group 2 as opposed to 1 case out of 54 (1.8\%) in Group 1; the difference resulted statistically significant $(p=<0.05)$ using the Fisher exact test (in consideration of the small sample size). When stratifying such 9 cases, a clear and direct correlation with size and grade was demonstrated but not with age and gender. Quite obviously, most (Group 1: 1/1, Group 2: 6/9) of these cases were of the CCRCC subtype. 


\section{Discussion}

RCC is a common tumor whose prognosis greatly depends on the time of diagnosis and on stage. Stage I-II tumors (i.e., intra-parenchymal tumors without extra-renal extension or nodal involvement) have a better prognosis than stage III tumors, which are by definition characterized by extra-renal extension or loco-regional lymph node metastases. Nevertheless, pathological staging, though based on apparently stringent criteria, [7] frequently is a complex issue, in view of inconstant inter-observer agreement [6] and the very variable patterns of invasiveness.

Microscopic assessment of extra-renal tumoral extension relies upon accurate and generous macroscopic sampling at critical sites $[6-8,16,17]$, and may be supported by immunohistochemistry, when deemed necessary.

To possibly overcome such troubles, we have experienced the more complete and standardized sampling technique of nephrectomies described herein, aimed at obtaining more accurate detection of intra-venous dissemination of the tumor, thus possibly improving inter-observer reproducibility.

Most of the results of the current study do not differ from those found in the literature, with particular regard to age, size, grade and histological subtypes. The minimal differences we detected did not show statistically significant differences, except for gender.

The main endpoint of this study was to evaluate whether or not the introduction of a new standardized sampling technique and the use of immunohistochemistry in doubtful cases, could facilitate the detection of neoplastic renal sinus invasion.

The results of our study seem to support such hypothesis, with specific reference to the infiltration of the intra-parenchymal branches of the renal vein, which could have previously been underestimated. In fact, while the overall number of pT3x cases is quite similar in both study groups, relevant differences were detected as to the sites of extra-renal invasion.

In principles, pT3a encompasses cases with fat (perinephric and renal sinus) invasion and intra-parenchymal veins invasion and, considering this parameter per se, we could not show relevant differences in the 2 investigated groups. Nevertheless, when taking into account individual sites of involvement, the occurrence of tumoral venous thrombosis, the main focus of our study, was more frequently detected in Group 2 and the difference was statistically significant.

Unfortunately, the relatively limited sample size and the restricted follow-up time do not allow for definitive conclusions, with specific regard to the prognostic implications of the proposed procedure. Nevertheless, taking into account the data of the literature, it seems likely that RCC patients could benefit of better fitting treatment, based on more accurate staging procedures, which would better disclose the occurrence of intra-renal veins tumoral thrombosis, thus avoiding inappropriate under-staging.

Author Contributions: Conceptualization, M.G.M. and M.M.; methodology, M.M., G.L. and G.I,; software, F.F.; validation, M.G.M., M.M. and MB; formal analysis, M.G.M. and F.F.; investigation, M.M., F.F., G.C. and V.R.; resources, G.L, M.B and G.I.; data curation, M.M. and F.F.; writing-original draft preparation, F.F. and M.G.M.; writing - review and editing, E.M.; visualization, F.F.; supervision, M.G.M., M.B. and E.M.; project administration, E.M.;. All authors have read and agreed to the published version of the manuscript.

Funding: This research received no external funding.

Informed Consent Statement: Informed consent was obtained from all subjects involved in the study.

Data Availability Statement: Data sharing not applicable. 
Conflicts of Interest: The authors declare no conflict of interest.

\section{References}

1. Moch, H.; Amin, M.B.; Argani, P.; Cheville, J.; Delahunt, B.; Martignoni, G.; Medeiros, L.J.; Srigley, J.R.; Tan, P.H.; Tickoo, S.K. Renal cell tumors. In WHO Classification of Tumors of the Urinary System and Male Genital Organs, 4th ed.; Moch, H., Humphrey, P.A., Ulbright, T.M., Reuter, V.E., Eds; International Agency for Research on Cancer: Lyon, France, 2016; pp. 11-76.

2. Rini, B.I.; McKiernan, J.M.; Chang, S.S.; Choueiri, T.K.; Kenney, P.A.; Landman, J.; Leibovich, B.C.; Tickoo, S.K.; Vikram, R.; Zhou, M.; et al. Kidney. In AJCC Cancer Staging Manual, 8th ed.; Amin, M.B., Ed; Springer, Switzerland, 2017, pp. 747-756.

3. Taneja, K, Williamson, SR. Updates in pathological staging and histologic grading of renal cell carcinoma. Surg Pathol Clin 2018, 11, 797-812.

4. Brierley, J.D.; Gospodarowicz, M.K.; Wittekind, C. Kidney. In TNM Classification of Malignant Tumours, 8th ed. Brierley, J.D.; Gospodarowicz, M.K.; Wittekind, C., Eds; UICC-Wiley Blackwell, Oxford, UK; 2017, pp. 199-201.

5. SEER cancer stat facts: kidney and renal pelvis cancer. SEER Cancer Statistics Review. National Cancer Institute. Bethesda, MD. Available online: http://seer.cancer.gov/statfacts/html/kidrp.html. (accessed on 30 December 2020).

6. Williamson, S.R.; Rao, P.; Hes, O.; Epstein, J.I.; Smith, S.C.; Picken, M.M.; Zhou, M.; Tretiakova, M.S.; Tickoo S.K.; Chen, Y.B.; et al. Challenges in Pathologic Staging of Renal Cell Carcinoma: A Study of Interobserver Variability Among Urologic Pathologists. Am J Surg Pathol 2018, 42, 1253-1261.

7. Trpkov, K.; Grignon, D.J.; Bonsib, S.M.; Amin, M.B.; Billis, A.; Lopez-Beltran, A.; Samaratunga, H.; Tamboli, P.; Delahunt, B.; Egevad, L.; et al. members of the ISUP Renal Tumor Panel. Handling and staging of renal cell carcinoma: the International Society of Urological Pathology Consensus (ISUP) conference recommendations. Am J Surg Pathol 2013, 37, $1505-1517$.

8. Bonert, M.; Kuo-Cheng, H.; Trpkov, K. Handling, sampling and stage evaluation of Renal Cell Carcinoma: A practical guide. Diagn Histopathol, 2016, 22, 57-67.

9. Thompson, R.H.; Blute, M.L.; Krambeck, A.E.; Lohse, C.M.; Magera, J.S.; Leibovich, B.C.; Kwon, E.D.; Frank, I.; Cheville, J.C. Patients with pT1 renal cell carcinoma who die from disease after nephrectomy may have unrecognized renal sinus fat invasion. Am J Surg Pathol. 2007, 31, 1089-1093.

10. Yoshida, T.; Ohe, C.; Tsuzuki, T.; Sugi, M.; Kinoshita, H.; Tsuta, K.; Matsuda T. Clinical impact of segmental renal vein invasion on recurrence in patients with clinical T1 renal cell carcinoma undergoing partial nephrectomy. Int J Clin Oncol. 2020, 25, 464-471.

11. Hatcher, P.A.; Anderson, E.E.; Paulson, D.F.; Carson, C.C.; Robertson, J.E. Surgical management and prognosis of renal cell carcinoma invading the vena cava. J Urol 1991, 145, 20-23.

12. Taneja, K.; Arora, S.; Rogers, C.G.; Gupta, N.S.; Williamson S.R. Pathological staging of renal cell carcinoma: a review of 300 consecutive cases with emphasis on retrograde venous invasion. Histopathology 2018, 73, 681-691.

13. Bonsib, S.M. The renal sinus is the principal invasive pathway: a prospective study of 100 renal cell carcinomas. Am J Surg Pathol. 2004, 28, 1594-1600.

14. Bonsib, S.M. Renal veins and venous extension in clear cell renal cell carcinoma. Mod Pathol 2007, 20, 44-53.

15. Bonsib, S.M.; Bhalodia, A. Retrograde venous invasion in renal cell carcinoma: a complication of sinus vein and main renal vein invasion. Mod Pathol 2011, 24, 1578-1585.

16. Bonsib, S.M. Macroscopic assessment, dissection protocols and histologic sampling strategy for renal cell carcinomas. Diagn Histopathol 2008, 14, 151-156.

17. Bonsib, S.M.; Bhalodia, A. Intravenous dissection, the optimum strategy for staging of renal cell carcinoma. Mod Pathol 2010, 23, $180 \mathrm{~A}$.

18. Houston Thompson, R.; Kurta, J.M.; Kaag, M. Tumor size is associated with malignant potential in renal cell carcinoma. J Urol. 2009, 181, 2033-2036.

19. Delahunt, B.; Cheville, J.C.; Martignoni, G.; Humphrey, P.A.; Magi-Galluzzi, C.; McKenney, J.; Egevad, L.; Algaba, F.; Moch, H.; Grignon, D.J. Members of the ISUP Renal Tumor Panel. The International Society of Urological Pathology (ISUP) grading system for renal cell carcinoma and other prognostic parameters. Am J Surg Pathol 2013, 37, 1490-1504. 